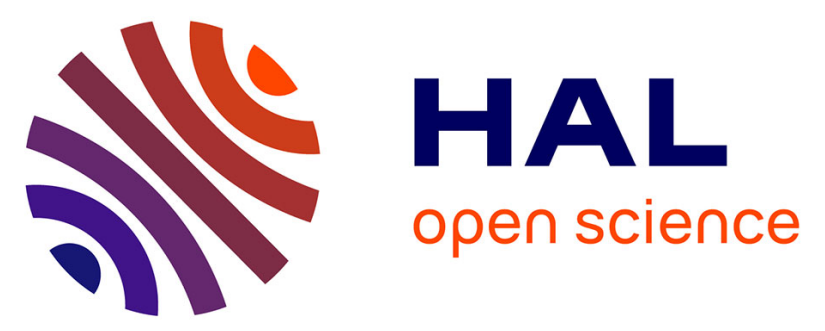

\title{
Diversification ou stabilité de la prédation au cours du Tardiglaciaire dans les Pyrénées françaises: et si on analysait les données?
}

Laure Fontana, Jacques Élie Brochier

\section{- To cite this version:}

Laure Fontana, Jacques Élie Brochier. Diversification ou stabilité de la prédation au cours du Tardiglaciaire dans les Pyrénées françaises: et si on analysait les données?. Bulletin de la Société préhistorique française, 2009, 106 (3), pp.477-490. 10.3406/bspf.2009.13871 . halshs-00408862

\section{HAL Id: halshs-00408862 \\ https://shs.hal.science/halshs-00408862}

Submitted on 26 May 2020

HAL is a multi-disciplinary open access archive for the deposit and dissemination of scientific research documents, whether they are published or not. The documents may come from teaching and research institutions in France or abroad, or from public or private research centers.
L'archive ouverte pluridisciplinaire HAL, est destinée au dépôt et à la diffusion de documents scientifiques de niveau recherche, publiés ou non, émanant des établissements d'enseignement et de recherche français ou étrangers, des laboratoires publics ou privés. 


\section{Laure FONTANA et Jacques Élie BROCHIER}

\section{Diversification ou stabilité de la prédation au cours du Tardiglaciaire dans les Pyrénées françaises : et si on analysait les données?}

\begin{abstract}
Résumé
Cet article présente les résultats de l'analyse de 56 séries fauniques issues de 21 sites archéologiques du Tardiglaciaire et du début du Postglaciaire. L'objectif était de tester l'hypothèse d'une diversification de la prédation à la fin du Tardiglaciaire dans les Pyrénées françaises. Les analyses effectuées démontrent que la date de $12300 \mathrm{BP}$ (non calibrée) marque une rupture dans le choix des espèces, le Renne n'étant plus chassé. Elles indiquent clairement qu'aucune diversification de la chasse aux grands mammifères n'est perceptible entre le Dryas ancien et le début du Postglaciaire. Enfin, elles mettent en évidence l'absence de toute augmentation significative de la part des petits gibiers capturés entre 15000 et $8000 \mathrm{BP}$, ce qui réfute l'opinion souvent émise d'une diversification de la prédation à la fin du Tardiglaciaire. Ces résultats ne sont pas sans rappeler ceux qui ont été obtenus pour le Jura français et les Alpes du nord.
\end{abstract}

\begin{abstract}
Results of the analysis of 56 faunal assemblages from 21 Lateglacial and Postglacial archaeological sites are presented in this paper. The aim of this study was to test the hypothesis of hunting diversification at the end of the Lateglacial in the French Pyrenees. The analysis demonstrates that a major change in animal hunting occurs at 12300 uncal. BP when Reindeer was no longer hunted. It also indicates that the diversity of hunted large mammals was the same from the Oldest Dryas to the onset of the Holocene. Finally, it reveals that there is no significant increase in the proportion of small game between 15000 uncal. BP and 8000 uncal. BP, which demonstrates that there was no hunting diversification at the end of the Lateglacial. Studies focusing on the French Jura and the Northern Alps have provided similar results.
\end{abstract}

La présence, dans des couches du Magdalénien supérieur et de l'Azilien, de restes plus ou moins nombreux d'Oiseaux, de Poissons et de Lagomorphes, a été interprétée par les préhistoriens français en termes de diversification des ressources animales. Ce changement majeur, caractérisé par l'intensification de la capture des petits gibiers et donc par l'«élargissement»de l'éventail des animaux chassés, est considéré soit comme une conséquence de la transformation des faunes à la fin du Tardiglaciaire, soit comme l'illustration de profondes mutations socio-économiques. Le tableau serait le suivant: des environnements plus fermés dans lesquels évoluaient des gibiers moins grégaires, des chasseurs armés en conséquence et 
nouvellement épris de petits animaux. Une telle image, tenace, subsiste en dépit des travaux menés sur les sites de l'Est de la France qui ont démontré l'absence de diversification de la subsistance (Bridault, 1994 et 1997). Il est vrai que les scénarios ont pu varier d'une région à l'autre, en fonction des contextes environnementaux, culturels et des ressources disponibles; encore faut-il le démontrer. Nous avons choisi la région pyrénéenne qui livre de nombreuses données pour la période considérée, qu'il s'agisse de faunes étudiées

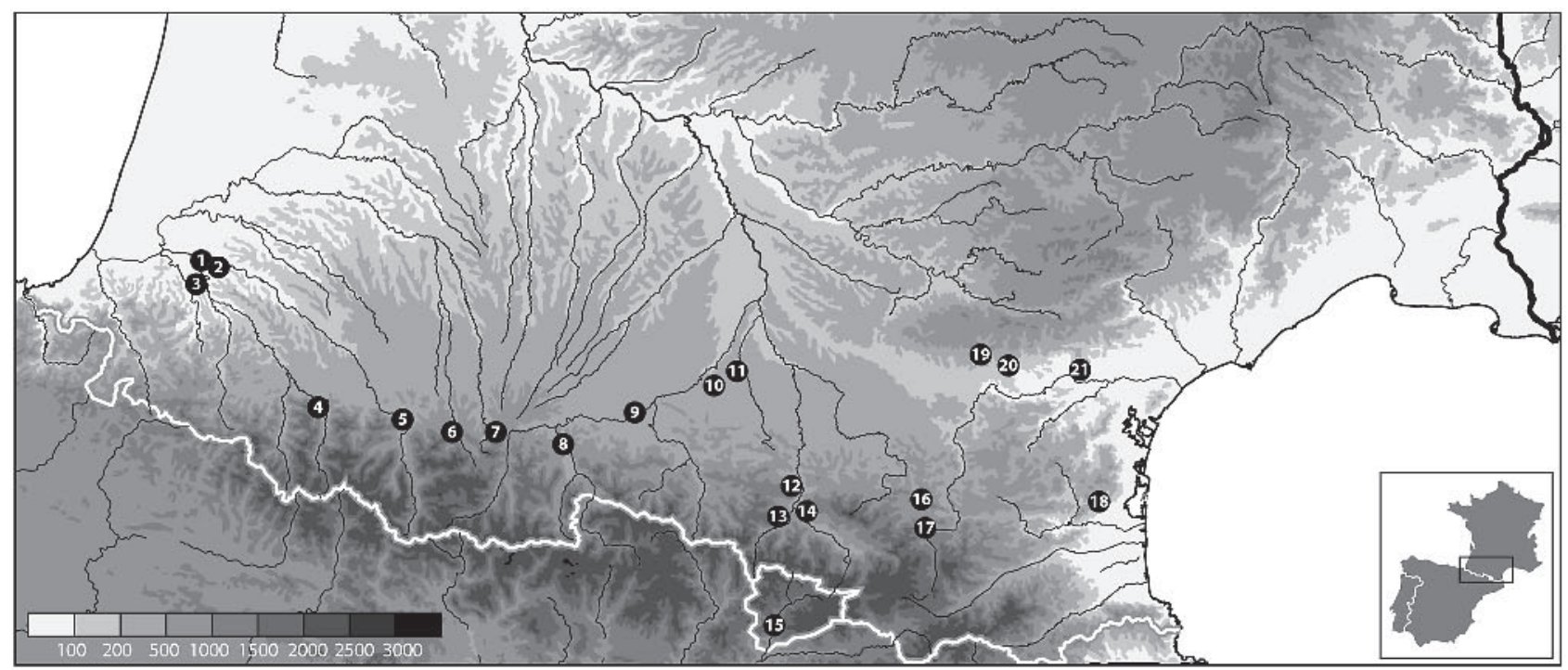

Fig. 1 - Carte des gisements cités dans le texte. 1 : Duruthy; 2 : Dufaure; 3 : Bourrouilla; $4:$ le Bignalats; 5 : les Espélugues; $6:$ Aurensan inf.; 7 : Espèche ; 8 : Troubat ; 9 : la Tourasse; 10 : Enlène; 11 : le Mas d'Azil ; 12 : Rhodes II; $13:$ la Vache; $14:$ les Églises; $15:$ la Margineda; $16:$ Belvis; 17 : Dourgne; 18 : les Conques; 19 : Canecaude I; 20 : Gazel; 21 : Tournal.

\begin{tabular}{|c|c|c|c|c|c|c|}
\hline $\mathbf{N}^{\circ}$ & Site & Sigle & Alt. & Commune & Couche & Référence des décomptes utilisés \\
\hline 1 & Duruthy & DR & 100 & Sorde-L'Abbaye (40) & $3,4,5$ & Delpech, 1978 et 1983 \\
\hline 2 & Dufaure & DUF & 100 & Sorde-L'Abbaye (40) & $\begin{array}{l}3 \mathrm{i}, 3 \mathrm{~s} \\
4 \mathrm{i}, 4 \mathrm{mi}, 4 \mathrm{~ms}, 4 \mathrm{~s}, 5\end{array}$ & $\begin{array}{l}\text { Altuna et Mariezkurrena, 1995; } \\
\text { Eastham, 1995; Le Gall, 1995a }\end{array}$ \\
\hline 3 & Bourrouilla & $\mathrm{BOU}$ & 51 & Arancou (64) & A, B1, B2 & $\begin{array}{l}\text { Fosse, 1999; Le Gall, 1999a; } \\
\text { Costamagno, 2004; Eastham, } 1998 \\
\text { et } 1999\end{array}$ \\
\hline 4 & Le Bignalats & $\mathrm{BIG}$ & 410 & Arudy (64) & Cbg-ns & Altuna et Marsan, 1986 \\
\hline 5 & Les Espélugues & ESPG & 430 & Lourdes (65) & locus I & Omnes, 1984; Clot, 1983 \\
\hline 6 & Aurensan inférieur & $\mathrm{AU}$ & 570 & Bagnères-de-Bigorre (65) & magdalénienne & Clot, 1983; Bouchud, 1972 \\
\hline 7 & Bois du Cantet & ESPE & 380 & Espèche (65) & magdalénienne & $\begin{array}{l}\text { Clot, 1984; Mourer-Chauviré, } \\
\text { in Clot, } 1984\end{array}$ \\
\hline 8 & Grotte-abri du Moulin & TB & 550 & Troubat (65) & $5 b, 5 c, 6 a, 6 b, 7 a$ & $\begin{array}{l}\text { Martin, 1994; Le Gall, 2003; } \\
\text { Costamagno, 2005; Laroulandie, } 2007\end{array}$ \\
\hline 9 & La Tourasse & TR & 279 & Saint-Martory (31) & D1 à D9 & Gourdain, 2000 \\
\hline 10 & Enlène, salle des Morts & ENL & 490 & Montesquieu-Avantès (09) & 3 et déblais & Delpech, 1981 \\
\hline 11 & Galerie rive droite & $A Z$ & 315 & Le Mas d'Azil (09) & magdalénienne & Patou, 1984; Vilette, 1983 \\
\hline 12 & Rhodes II & $\mathrm{RHO}$ & 520 & Arignac (09) & $5,6,7$ & Delpech, 1983 \\
\hline 13 & La Vache & VA & 595 & Alliat (09) & $1,2,3,4$ & $\begin{array}{l}\text { Pailhaugue, } 1995 \text { et } 2003 \text {; } \\
\text { Laroulandie, } 2000\end{array}$ \\
\hline 14 & Les Églises & EGL & 550 & Ussat (09) & $\begin{array}{l}3,4,6,8,8 b \\
9,9 b, 11\end{array}$ & $\begin{array}{l}\text { Delpech et Le Gall, 1983; } \\
\text { Laroulandie, } 1998\end{array}$ \\
\hline 15 & La Balma Margineda & MARG & 970 & Sant Julià de Lòria (And.) & $4,5,6,7,8$ & $\begin{array}{l}\text { Le Gall 1995b, } 1999 b \text { et } 2008 ; \\
\text { Gardeisen, } 2008 \text { et sous presse }\end{array}$ \\
\hline 16 & Belvis & BEL & 960 & Belvis (11) & $1,2,3,4$ & $\begin{array}{l}\text { Fontana, 1998a et 1999; Vilette, } 1983 \\
\text { et 1994; Le Gall, 1999b }\end{array}$ \\
\hline 17 & Dourgne & DOU & 710 & Fontanès-de-Sault (11) & 9 & Geddes, 1993 \\
\hline 18 & Les Conques & $\mathrm{CO}$ & 240 & Vingrau (66) & 2 et 3 & Moigne, 2003 et inédit \\
\hline 19 & Canecaude I & CAN & 250 & Villardonnel (11) & II & $\begin{array}{l}\text { Fontana, } 1998 b \text { et } 1999 \text {; Vilette, } 1983 \text {; } \\
\text { Le Gall, } 1999 b\end{array}$ \\
\hline 20 & Gazel & GZ & 200 & Sallèles-Cabardès (11) & 7,7 bis, $7 / 8,8$ & $\begin{array}{l}\text { Fontana, } 1998 b \text { et } 1999 \text {; Vilette, 1983; } \\
\text { Desse-Berset, inédit }\end{array}$ \\
\hline 21 & Tournal & TG & 100 & Bize-Minervois (11) & IVA-G & Patou-Mathis et al., 1999; Vilette, 1983 \\
\hline
\end{tabular}

Tabl. 1 - Les sites étudiés : provenance des échantillons et références bibliographiques. 


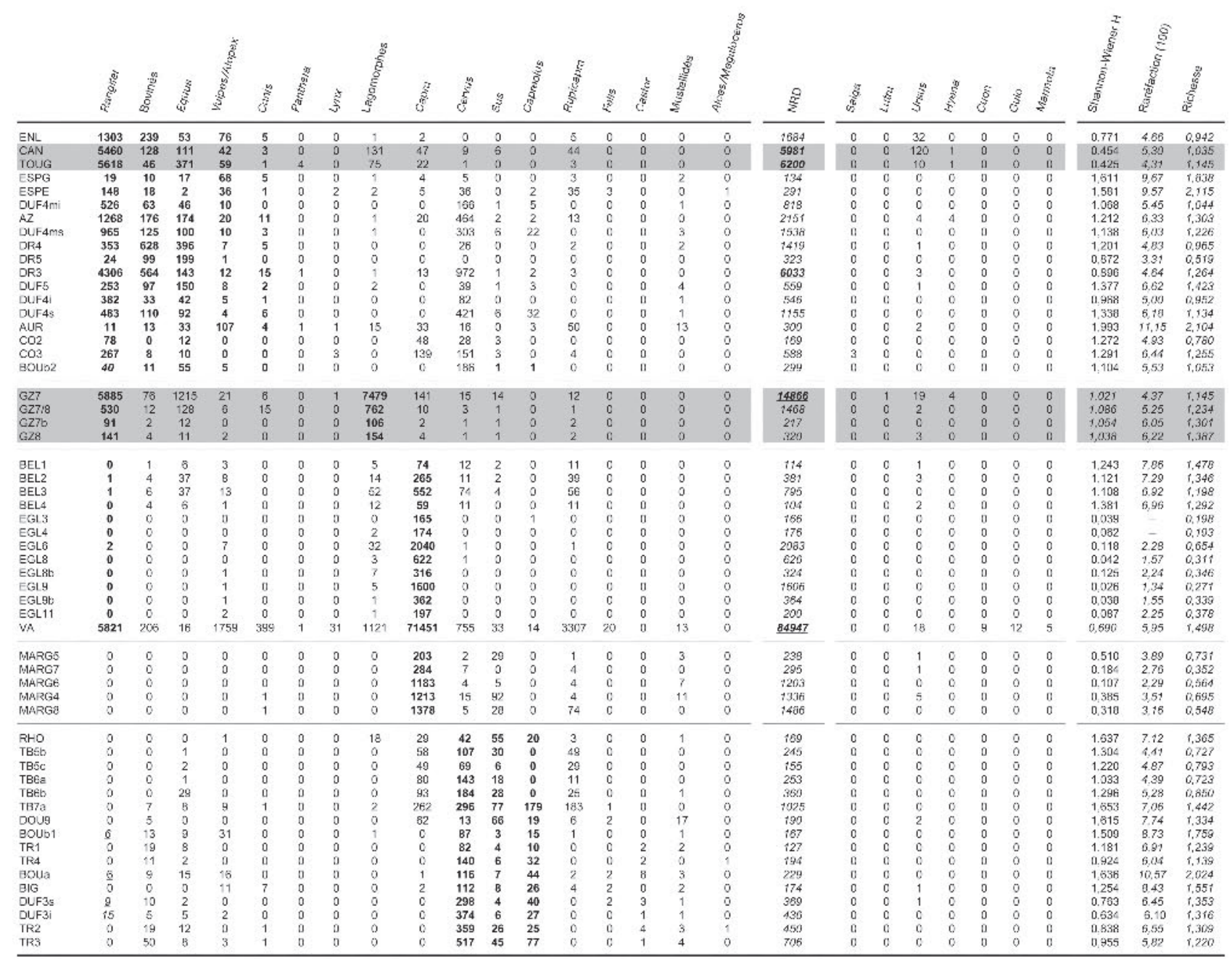

Tabl. 2 - Tableau de contingence croisant les échantillons et les taxons de grands mammifères. Shannon-Wiener H : indice de diversité de ShannonWiener; Raréfaction (100) : nombre normalisé de taxons pour 100 restes; Richesse : indice de richesse de Margalef. Les sites en grisé sont ceux qui se situent hors de la chaîne pyrénéenne.

ou encore de dates radiocarbone. Nos travaux antérieurs, fondés sur les données des sites de la partie orientale des Pyrénées, avaient déjà permis de proposer certaines hypothèses (Fontana, 1998b et 1999; Bridault et Fontana, 2003) que seule l'analyse détaillée de données provenant de l'ensemble du massif pouvait éventuellement valider.

Notre premier objectif est de préciser les modalités de la modification des faunes chassées qui a lieu entre 15000 et $8700 \mathrm{BP}$ afin de mettre en évidence un éventuel changement et de situer les séries par rapport à cet événement en caractérisant les associations. Le calcul de la diversité des spectres de mammifères chassés (ongulés, carnivores et lagomorphes), répartis en deux groupes chronologiques, permet alors de discuter d'une éventuelle diversification des ressources. Il n'est pas question ici de commenter certaines tendances en termes de modèles socio-économiques («économie de disette» ou «d'abondance», influence du milieu naturel sur les choix humains). L'enjeu de cette étude est, plus modestement, de tester l'hypothèse d'une diversification à partir de l'analyse des données issues de
56 niveaux archéologiques de 21 sites des Pyrénées françaises (fig. 1 et tabl. 1), retenus à l'issue de l'analyse critique des échantillons (taille de la collection, fiabilité de la stratigraphie et/ou de l'attribution chronologique $)^{1}$. Les données ont été traitées par unité stratigraphique, sans tenir compte des regroupements de couches parfois proposés. Seul le site de la Vache fait exception à la règle, puisque les restes fauniques de la salle Monique ont été étudiés comme provenant d'un même ensemble, l'archéozoologue n'ayant pas tenu compte du découpage artificiel des $80 \mathrm{~cm}$ de remplissage en quatre unités effectué par les fouilleurs (Clottes et Delporte, 2003). Ainsi, 56 séries ont été analysées à partir de l'enregistrement des fréquences absolues de 24 taxons de mammifères (tabl. 2). Nous avons cependant réduit ce nombre à 17 en raison du caractère exceptionnel de certaines occurrences (Saïga, Lutra, Cuon, Gulo, Marmota) ${ }^{2}$ ou de l'origine, vraisemblablement naturelle, des restes de certaines espèces (Ours et Hyène). Enfin, la qualité de l'information disponible nous a contraints à effectuer certains regroupements d'espèces (cf. tabl. 2). 


\section{MODIFICATION DES FAUNES CHASSÉES ENTRE 15000 ET $8000 \mathrm{BP}$}

Décrire objectivement et comprendre les structures sous-jacentes à un tableau de contingence de la taille du tableau 2 n'est guère réalisable sans avoir recours aux traitements factoriels. Dans une analyse qui se veut intrinsèque, nous tentons de comprendre les modifications dans le choix des gibiers au cours du temps, dans un référentiel constitué des sites pyrénéens du Paléolithique supérieur récent et du Mésolithique. Il est inutile, et méthodologiquement très contestable, d'attribuer une signification environnementale aux différents taxons et de constituer des groupes «écologiques» $a$ priori, ce que les résultats des analyses isotopiques ont d'ailleurs confirmé (Drucker, 2001). L'éventuelle information de nature environnementale est naturellement contenue dans le tableau de données et c'est à l'analyse, ici l'analyse factorielle des correspondances simples, de la mettre en évidence.

Dans le plan des deux premiers axes factoriels de la figure 2A, une série d'échantillons d'âges variés caractérisés par l'abondance des bouquetins (Belvis, la Vache, la Balma de la Margineda, les Églises) s'oppose sur le premier axe à tous les autres, dont la position sur le deuxième axe est fonction du poids qu'y occupent les Lagomorphes (côté positif), le Cerf (Cervus elaphus) et le Chevreuil (Capreolus capreolus) (côté négatif). À ce stade, l'axe 2 trie donc les échantillons suivant la plus ou moins grande ouverture du milieu. L'élimination du premier groupe, caractérisé par de fortes fréquences relatives de bouquetins, permet de préciser la structure qui apparaissait précédemment sur l'axe 2. En effet, sur le premier axe, les descripteurs qui caractérisent les milieux ouverts froids sont opposés à ceux qui caractérisent les milieux tempérés plus fermés (fig. 2B), ce qui, pour la courte tranche chronologique concernée par cette étude, revient à attribuer un sens grossièrement chronologique à cet axe. Une fois encore, un groupe d'échantillons très originaux (Gazel), caractérisés par l'abondance des Lagomorphes, ici des lièvres variables, s'isole dans le troisième quadrant.

Les nombreuses datations radiocarbone disponibles (fig. 3), de qualité très inégale, indiquent que la date 12300 BP (non calibrée) sépare deux groupes d'échantillons (cf. fig. 2B) : les échantillons, que nous qualifierons par la suite d' «anciens», dans lesquels le Renne (Rangifer tarandus) est présent et les échantillons «récents » dans lesquels il est absent. Dans ce référentiel, la date radiocarbone $12300 \mathrm{BP}$ (non calibrée) marque donc la disparition du Renne, un constat qui n'a rien de neuf. Quelques séries, cependant, semblent en contradiction avec cette conclusion : celles de l'abri Dufaure (couches 3 et 4), de Bourrouilla (couches B1 et A) et de l'abri Duruthy (couche 3). En effet, sur la figure 2C (dérivée de la figure 2B) où les échantillons sont différenciés selon l'abondance relative des restes de Renne, on constate que quelques-uns sont positionnés dans le groupe «anciens» alors que leur âge radiocarbone est postérieur à 12300 BP (Duruthy 3, Dufaure 4s), alors que d'autres, qui livrent quelques restes de Renne, se placent dans le groupe des échantillons «récents» (Dufaure 3). Suffisent-ils à mettre en doute le scénario proposé de disparition, à la fois rapide et synchrone, du Renne sur une grande partie du territoire français (Fontana, 1998a ; Bridault et al., 2000) ou valident-ils l'existence de refuges, septentrionaux et/ou altitudinaux, jusque vers 10900 BP (Dufaure 4s) ? Analysons de plus près les données chronologiques et contextuelles. La date obtenue à Duruthy pour le sommet de la couche 3 est $11150 \pm 220 \mathrm{BP}$ (LY 858). Elle est obtenue par mesure de l'activité ${ }^{14} \mathrm{C}$ du collagène extrait d'une grande quantité d'ossements non déterminés spécifiquement (Évin, 1978). Ce résultat signifie qu'il n'y a qu'un peu plus de 4 chances sur 100 pour que l'âge radiocarbone réel dépasse $11590 \mathrm{BP}$, donc très loin du seuil de $12300 \mathrm{BP}^{3}$. Avec 4306 restes de Renne pour un total de 6033 restes déterminés $(71,4 \%)$, nous ne pouvons invoquer d'éventuelles perturbations qui auraient dispersé quelques restes dans un horizon d'âge récent. Le matériel archéologique de cette couche, surmontée, rappelons-le, par un niveau azilien (couche 2), est d'autre part rapporté sans ambiguïté au Magdalénien final à harpons. Enfin, il ne faut pas oublier que la date LY 858 concerne le sommet de l'épaisse $(50 \mathrm{~cm})$ couche 3 que rien ne permet, bien au contraire (Thibault, 1978, p. 93 ; Paquereau, 1978), de considérer comme un dépôt rapide. En l'attente de datations SMA sur ossements de Renne de la couche 2 et des différents niveaux de la couche 3, assorties de dosages du carbone et de l'azote, il paraît plus raisonnable d'assigner à l'essentiel du matériel archéologique de la couche 3 de l'abri Duruthy un âge supérieur ou égal à 12300 BP. Le cas de l'abri Dufaure est très différent. Le Renne occupe une place importante, bien que régulièrement décroissante, dans les spectres de la couche 4 (70 \% à la base, $42 \%$ au sommet). Les datations radiométriques de cette couche sont compatibles, compte tenu des écart-types, avec un seuil à 12300 BP. La date LY 2666 de la couche 4s fait cependant exception, comme les dates radiocarbone des différents niveaux de la couche 3 (3i, $3 \mathrm{~m}$ et $3 \mathrm{~s}$ ), qui fixent sa mise en place au début du Postglaciaire. Il y a certainement lieu d'être très circonspect dans l'utilisation de ces dates. En effet, les taux de collagène conservé dans les os de la couche 3 sont infimes $(0,45 \%$ en moyenne), trois fois moindres que dans les os de la couche de base (couche 6). Les résultats du dosage du carbone et de l'azote effectués par T. Stafford et publiés par J. Évin (1995) indiquent que l'excès de carbone (Bocherens et al., 2005) dans un des échantillons de la couche 6 n'est que de $0,54 \%$ et donc que les dates de cette unité sont plausibles. Mais qu'en est-il des valeurs du carbone et de l'azote des ossements manifestement très altérés (Straus, 1995, p. 75) de la couche 3 ? Encore une fois, les données analytiques manquent pour valider de telles dates obtenues à partir du collagène extrait de plusieurs centaines de grammes d'ossements non déterminés spécifiquement ou sur des extraits d'acides aminés provenant d'un os indéterminé. Quoi qu'il en 

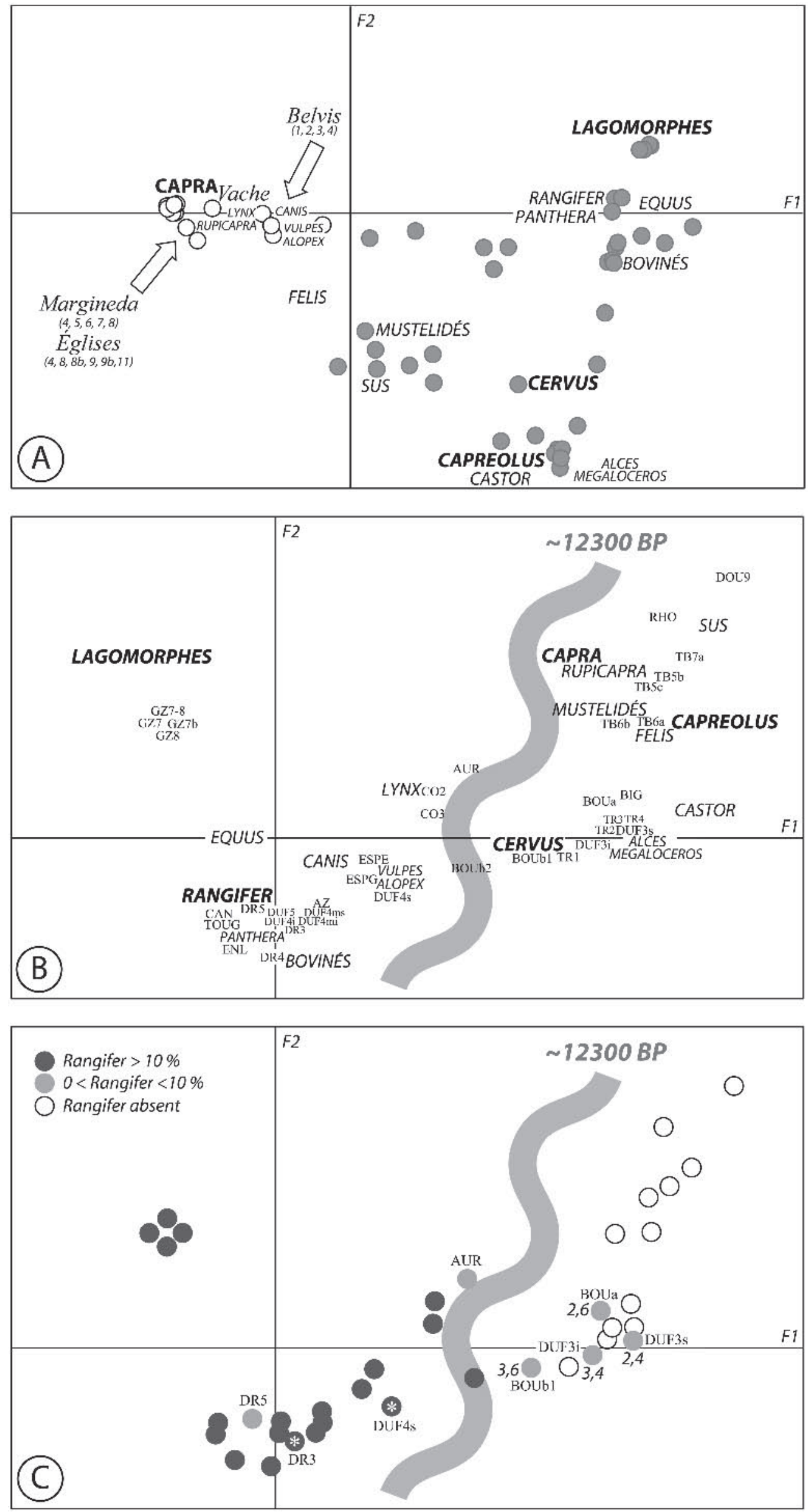

Abréviations utilisées :

- GZ : Gazel;

- CAN : Canecaude;

- ENL : Enlène

- DR : Duruthy;

- DUF : Dufaure;

- AZ : Mas d'Azil

- ESPE : Espèche;

- ESPG : Espélugues

- CO : Conques;

- AUR : Aurensan;

- BOU : Bourrouilla;

- TR : Tourasse;

- BIG : le Bignalats;

- TB : Troubat;

- RHO : Rhodes II;

- DOU : Dourgne.

A. Plan factoriel 1/2 obtenu à partir du tableau complet. Seuls les spectres à Bouquetin dominant ou abondant, opposés sur le premier axe à tous les autres (ronds gris), sont nommés. Les taxons qui contribuent le plus largement au premier axe factoriel sont en gras.

B. Plan factoriel $1 / 2$ obtenu après élimination des spectres dans lesquels le Bouquetin est dominant (Balma de la Margineda, grotte des Églises et grotte de Belvis). La ligne grise ondulée sépare les échantillons anciens dans lesquels le Renne est présent de ceux qui livrent une faune de type actuel.

C. Même plan factoriel sur lequel apparaissent seulement les spectres, répartis selon trois classes de fréquence des restes de Renne. Les nombres associés aux ronds gris sont les fréquences relatives des restes de Renne. Les ronds noirs étoilés désignent les deux échantillons d'âge récent à forte fréquence relative de Renne.

Fig. 2 - Analyses factorielles des correspondances du tableau de contingence croisant taxons et échantillons. 


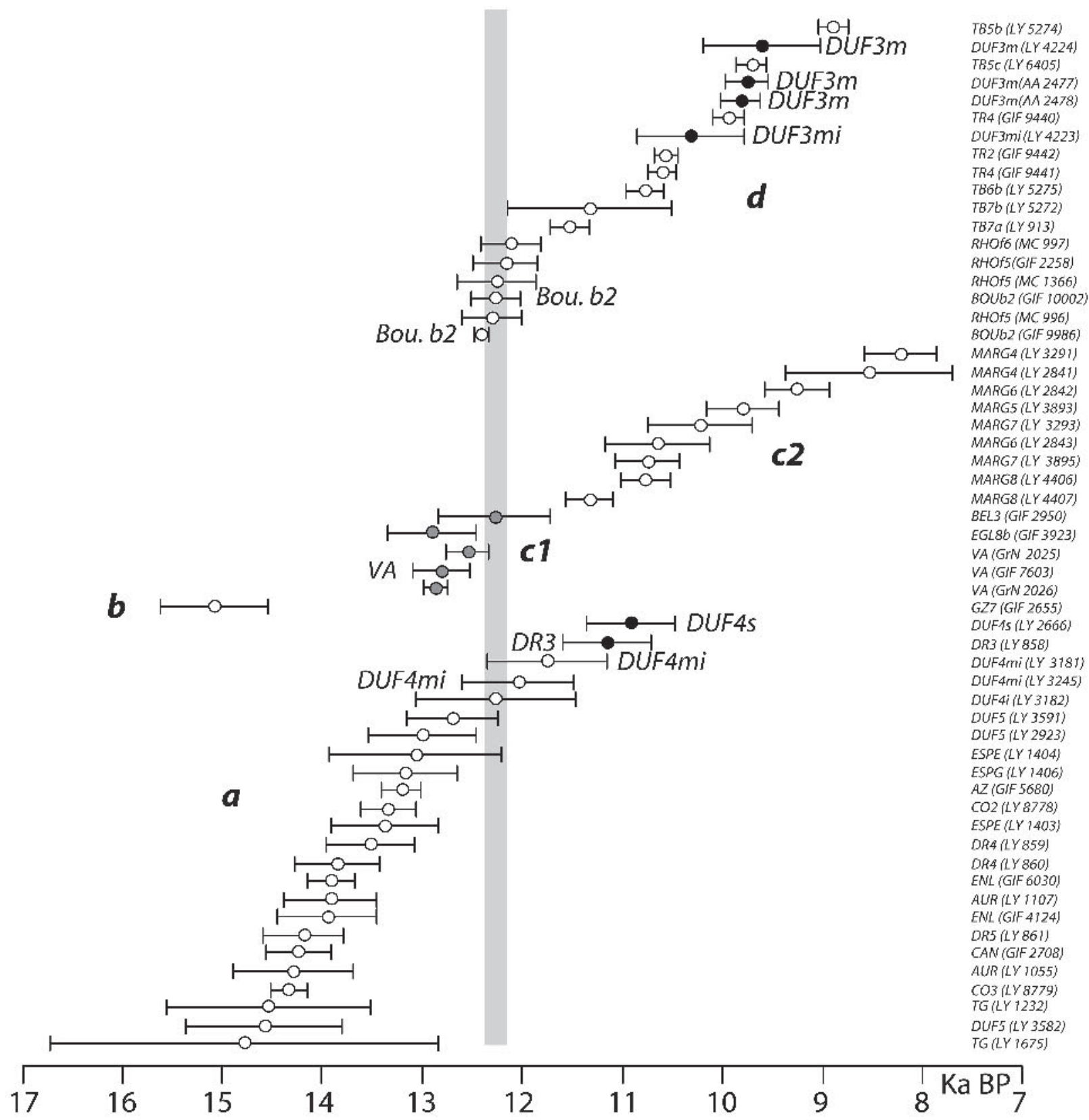

Fig. 3 - Dates radiocarbone des échantillons fauniques analysés regroupées suivant les ensembles définis par les analyses factorielles. a : phase ancienne à Renne; $\mathbf{b}$ : phase ancienne à Lagomorphes; $\mathbf{c 1}$ et $\mathbf{c 2}:$ phases ancienne et récente à Bouquetin; $\mathbf{d}:$ phase récente.

soit de l'âge réel des différents horizons aziliens de la couche 3, la présence de quelques restes de Renne ( 9 en 3 sup., 15 en 3 inf.) relève plus vraisemblablement, dans ce contexte sédimentaire, d'un remaniement (phénomène normal dans les dépôts de grotte et d'abri-sous-roche) à partir du sommet de la couche 4 . C'est du moins la position que nous prendrons en l'attente de datations SMA de restes déterminés spécifiquement. La présence de quelques restes de Renne dans les couches B1 et A de la grotte du Bourrouilla devrait, en l'absence de datations spécifiques, s'interpréter de la même façon. En effet, les échantillons de ces deux couches sont postérieurs à $12300 \mathrm{BP}$, comme l'indiquent les dates de la couche B2 sous jacente et l'importance du Chevreuil dans le spectre faunique.
Les données fiables disponibles indiquent donc qu'à partir de 12300 BP, le Renne ne fait plus partie des espèces chassées. Cette date marque une rupture dans les cortèges fauniques qui s'est également traduite par l'augmentation, plus ou moins importante selon les secteurs géographiques, de la part du Cerf. Néanmoins, rappelons que ce gibier était déjà bien représenté dans les spectres dès 13000 BP (Fontana, 1998a; Bridault et Fontana, 2003), au Mas d'Azil, à Espèche, à Duruthy $(\mathrm{C} 3)$ et à Dufaure $(\mathrm{C} 4)$, avant de devenir, vers $12300 \mathrm{BP}$, le premier gibier chassé dans ce secteur occidental de basse altitude (Bourrouilla B2). Notons qu'un tel choix du Cerf au Bourrouilla dès 1240012300 BP, tout en démontrant le développement d'un milieu favorable à l'espèce, constitue un argument 
supplémentaire qui va à l'encontre de l'existence d'une zone de maintien du Renne dans ce secteur occidental. Le développement de la chasse au Cerf dès 13000 BP se poursuit après 12300 BP et il n'est pas doublé d'une forte augmentation de la part du Chevreuil, dont les restes n'excèdent les $12 \%$ qu'à Troubat $7 \mathrm{a}(17 \%)$. Il en est globalement de même pour le Sanglier (Sus scrofa), à deux exceptions près puisqu'il est le premier gibier à Rhodes II $(33 \%)$ et à Dourgne $(39 \%)$, sites où la chasse fut très diversifiée (cf. tabl. 2). Ceci s'explique par la part importante du Bouquetin (Capra pyrenaïca) et dans une moindre mesure du Chamois (Rupicapra pyrenaïca) dans les sites postérieurs à $12300 \mathrm{BP}$ des Pyrénées centrales et orientales. Ainsi, les spectres de la période 12000 $8500 \mathrm{BP}$ de ce secteur pyrénéen sont-ils très proches de ceux de l'Italie du Nord-Est (données dans Bridault et Fontana, 2003).

\section{DIVERSITÉ DES SPECTRES FAUNIQUES ET CHRONOLOGIE}

La diversité des spectres fauniques serait-elle différente selon la période considérée? Il suffit d'ouvrir un manuel d'écologie numérique (Daget, 1976; Legendre et Legendre, 1984 et 1998; Magurran, 1988 par exemple) ou d'analyse paléoécologique (Hammer et Harper, 2006) pour prendre conscience de la complexité des concepts de diversité et de richesse, et de la multiplicité des solutions mathématiques proposées; une raison qui devrait être amplement suffisante pour que, dans chaque étude, la méthode de mesure de la richesse ou de la diversité choisie soit clairement précisée, et ce d'autant que certains indices ne peuvent être utilisés en contexte archéologique. C'est le cas, par exemple, de la régularité (ou l'équitabilité pour rester francophone), une mesure proposée par E.C. Pielou (Pielou, 1966) à partir de l'entropie de Shannon-Wiener (Shannon et Weaver, 1949; Spellerberg et Fedor, 2003) qu'il est illicite d'utiliser lorsque le nombre réel de taxons est inconnu (Peet, 1974) ${ }^{4}$ et qu'il ne faudrait pas confondre avec «l'indice de Shannon». La régularité mesurée à partir d'échantillons prélevés en contexte archéologique (de taille toujours trop faible pour que le nombre réel de taxons ne soit même qu'approché) est ainsi à la fois très instable et systématiquement surévaluée. Nous quantifierons donc la diversité des différents échantillons à l'aide de l'entropie de Shannon-Wiener, beaucoup moins dépendante du nombre réel de taxons. Si la richesse spécifique d'un échantillon et sa diversité sont des paramètres descriptifs très importants, l'archéozoologue est contraint de les estimer d'une façon qui n'est guère licite car, en toute rigueur, seul le nombre d'individus, qui n'est pas le NMI, devrait être utilisé. Nous ne disposons cependant le plus souvent que des seuls décomptes en NR dont la relation avec le nombre minimal d'individus varie d'une série à l'autre en fonction des espèces représentées, de leur proportion respective et de la fragmentation différentielle. Néanmoins, si tous les indices proposés par les écologues et utilisés par les archéozoologues ${ }^{5}$ comportent ces biais les plus évidents, ils constituent le moins mauvais des moyens pour estimer la richesse et la diversité taxonomiques d'un échantillon. Ceci étant précisé, ajoutons que pour savoir si la diversité des animaux chassés dans les Pyrénées a évolué entre le Tardiglaciaire et le début du Postglaciaire, notamment au profit des petits gibiers, l'analyse des données doit naturellement prendre en compte l'ensemble des mammifères capturés, pas seulement les ongulés (par exemple Bridault, 1994 et 1997).

Dans notre échantillon, aucune différence significative n'existe entre la richesse, mesurée par l'indice de Margalef, des séries antérieures et celle des séries postérieures à $12300 \mathrm{BP}(\mathrm{F}, \mathrm{p}=0,391 ; \mathrm{t}, \mathrm{p}=0,719$, cf. tabl. 2). Ceci est vrai, que l'on considère l'ensemble des séries ou seulement celles dans lesquelles le Bouquetin n'est pas le gibier principal ${ }^{6}$. Quant à la

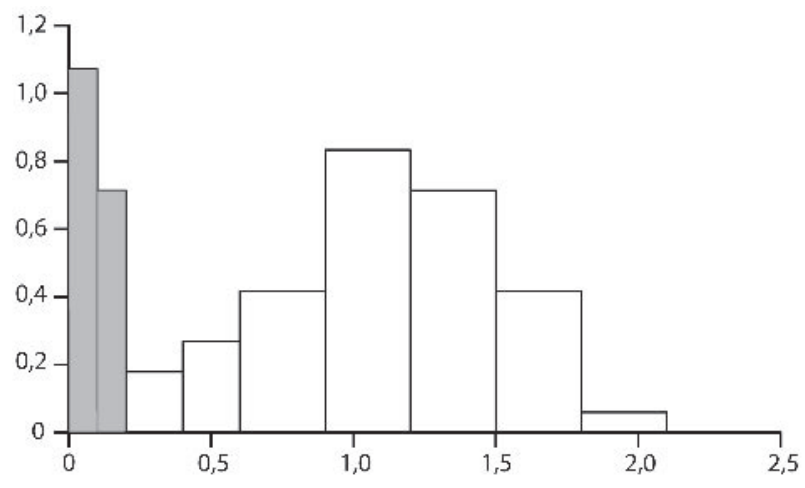

Fig. 4 - Distribution de l'entropie de Shannon-Wiener (en densité de fréquences) des 56 échantillons analysés. Dans ce référentiel, la valeur 0,20 est choisie comme limite supérieure des valeurs de faible diversité (en grisé).

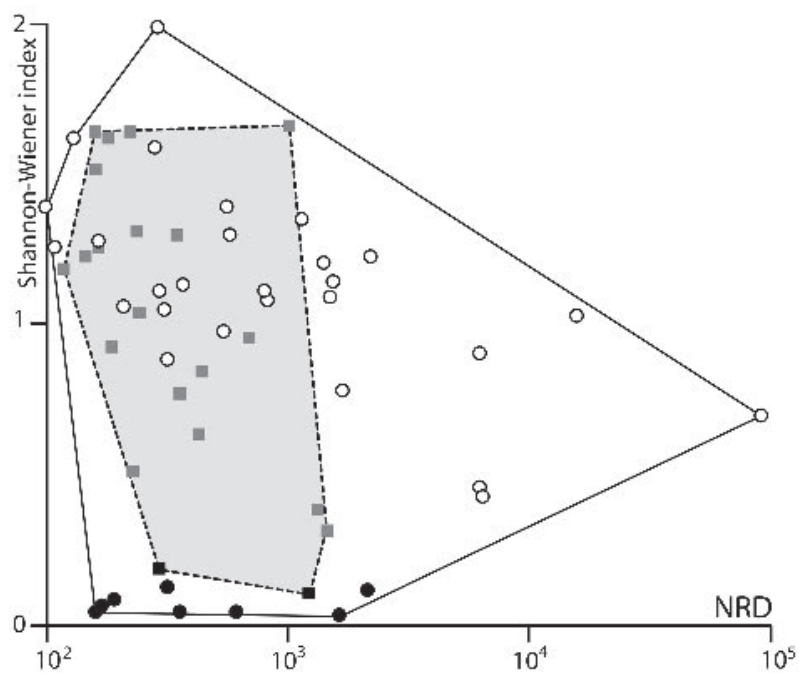

Fig. 5 - Relation entre diversité et NRD. Les deux polygones convexes délimitent les domaines des échantillons à Renne, antérieurs à $12300 \mathrm{BP}$ (ronds, trait continu), et des échantillons sans Renne, postérieurs à 12300 BP (carrés, trait tireté), tels qu'ils ont été définis par les analyses factorielles des correspondances et les classifications ascendantes hiérarchiques. Les symboles noirs représentent la position des échantillons à diversité faible $(\leq 0,20)$. L'échelle de l'axe des NRD est logarithmique. 
mesure de la diversité (fig. 4), il n'existe aucune différence significative, du point de vue des valeurs médianes et des distributions de l'entropie de ShannonWiener, entre les deux groupes chronologiques ${ }^{7}$ (fig. 5). Il n'existe donc pas de relation entre la diversité des spectres et leur position chronologique. Seraitil néanmoins possible que cette conclusion soit biaisée par l'existence d'un lien entre la taille des échantillons et leur diversité ? Il serait fort curieux qu'apparaisse dans les études écologiques un tel lien, qui ôterait, de fait, toute valeur aux différents estimateurs utilisés. En revanche, en contexte anthropique, un lien entre ces deux variables pourrait fort bien advenir, comme en témoigne, par exemple, la figure 2 de Costamagno et al., 2008: la diversité des échantillons y apparaît d'autant plus faible que leur taille est importante. Qu'en est-il? Dans le référentiel que nous analysons, aucun lien fort n'apparaît entre la diversité et le nombre de restes déterminés, que l'on considère l'ensemble des échantillons ( $\left.\mathrm{p}=0,0094 ; \mathrm{r}^{2}=0,12\right)$ ou, séparément, les deux groupes chronologiques isolés par l'analyse (cf. fig. 4). Dans le cas des échantillons dont l'âge est antérieur à $12300 \mathrm{BP}$, la liaison entre la diversité et le logarithme des NRD n'est pas significative $(\mathrm{p}=0,141)$; dans le cas des échantillons récents, la corrélation, significative $(p=0,010)$, reste faible. Le coefficient de détermination $\left(\mathrm{r}^{2}\right.$ de Spearman) ne vaut que 0,29 ; autrement dit, le modèle linéaire liant la diversité et le logarithme du NRD n'explique que $29 \%$ de la variance de l'une des deux variables lorsque l'autre est fixée. La taille des échantillons n'influe donc pas ou peu sur leur diversité. Lorsqu'une corrélation - négative - entre ces deux variables apparaît, elle provient de l'utilisation conjointe d'échantillons de très petite taille, nécessairement pauvres en taxons, et d'échantillons artificiels de très grande taille (matériel provenant de plusieurs unités archéologiques), donc plus riches en taxons.

\begin{tabular}{|c|c|c|c|c|c|c|c|c|c|c|}
\hline \multirow[b]{3}{*}{$15000 \mathrm{BP}$} & \multicolumn{6}{|c|}{ GRANDS MAMMIFĖRES (GM) } & \multicolumn{3}{|c|}{ OISEAUX (OI) } & \multirow{3}{*}{$\begin{array}{c}\text { POISSONS } \\
\text { NR } \\
\end{array}$} \\
\hline & \multirow{2}{*}{$\begin{array}{c}\text { Ongulés } \\
\text { NR }\end{array}$} & \multicolumn{2}{|c|}{ Lagomorphes } & \multicolumn{2}{|c|}{ Carnivores } & \multirow{2}{*}{$\begin{array}{c}\text { total } \\
N R \\
\end{array}$} & \multicolumn{3}{|r|}{ total } & \\
\hline & & NR & $\%$ & NR & $\%$ & & NR/NMI & $\%$ & $\mathrm{GM}+\mathrm{OI}$ & \\
\hline Canecaude I, C. II & 5805 & 131 & 2,2 & $45(42)$ & 0,8 & 5891 & NMI 4 & - & - & 2 \\
\hline Gazel C. 7 & 7358 & 7479 & 50,3 & $28(21)$ & 0,2 & 14865 & NMI 46 & - & - & 44 \\
\hline Tournal, C. IVA-G & 6062 & 75 & 1,2 & $64(59)$ & 1,0 & 6201 & NMI 17 & - & - & 1 \\
\hline Les Conques, C. 3 & 585 & 0 & 0,0 & $3(0)$ & 0,5 & 588 & 0 & 0,0 & 588 & 0 \\
\hline Aurensan inférieur & 159 & 15 & 5,0 & $126(107)$ & 42,0 & 300 & 45 & 13,0 & 345 & 1 \\
\hline Duruthy, C. 5 & 322 & 0 & 0,0 & $1(1)$ & 0,3 & 323 & 0 & 0,0 & 323 & 0 \\
\hline Duruthy, C. 4 & 1405 & 0 & 0,0 & $14(7)$ & 1,0 & 1419 & 5 & 0,4 & 1424 & 0 \\
\hline Duruthy, C. 3 & 6004 & 1 & 0,0 & $28(12)$ & 0,5 & 6033 & 127 & 2,1 & 6160 & 52 \\
\hline Enlène, C. 3 & 1602 & 1 & 0,1 & $81(76)$ & 4,8 & 1684 & 0 & 0,0 & 1684 & 0 \\
\hline Espèche & 247 & 2 & 0,7 & $42(36)$ & 14,4 & 291 & NMI 34 & - & - & 0 \\
\hline Les Conques, C. 2 & 169 & 0 & 0,0 & 0 & 0,0 & 169 & 0 & 0,0 & 169 & 0 \\
\hline Mas d'Azil & 2119 & 1 & 0,1 & $31(20)$ & 1,4 & 2151 & NMI 47 & - & - & - \\
\hline Les Espélugues, Loc. I & 58 & 1 & 0,8 & $75(68)$ & 56,0 & 134 & 0 & 0,0 & 134 & 1 \\
\hline Dufaure, C. 5 & 543 & 2 & 0,4 & $14(8)$ & 2,5 & 559 & 23 & 4,0 & 582 & 1 \\
\hline Dufaure, C. $4 \mathrm{~m}$ & 2328 & 1 & 0,0 & $27(20)$ & 1,2 & 2356 & 98 & 4,0 & 2454 & $2(\mathrm{C} 4)$ \\
\hline Dufaure, C. $4 \mathrm{i}$ & 539 & 0 & 0,0 & $7(5)$ & 1,3 & 546 & 14 & 2,5 & 560 & 0 \\
\hline Dufaure, C. $4 \mathrm{~s}$ & 1144 & 0 & 0,0 & $11(4)$ & 1,0 & 1155 & 21 & 1,8 & 1176 & 0 \\
\hline Les Églises, C. 3 à 11 & 5472 & 51 & 0,9 & $12(12)$ & 0,2 & 5535 & 1600 & 22,4 & 7135 & 502 \\
\hline Belvis, C. 1 à 4 & 1286 & 83 & 6,0 & $25(25)$ & 1,8 & 1394 & NMI 57 & - & - & 37 \\
\hline La Vache, C. 1 à 4 & 81603 & 1121 & 1,3 & 2244 & 2,6 & 84968 & 54724 & 39,2 & 139692 & - \\
\hline Arancou B2 & 294 & 0 & 0,0 & $5(5)$ & 1,7 & 299 & 15 & 314 & 4,8 & 1 \\
\hline \multicolumn{11}{|l|}{$12300 \mathrm{BP}$} \\
\hline Rhodes II, C. 5 à 7 & 149 & 18 & 10,7 & $2(1)$ & 1,2 & 169 & 7 & 176 & 4,0 & - \\
\hline Arancou B1 & 134 & 1 & 0,6 & $32(31)$ & 19,2 & 167 & - & - & - & - \\
\hline Arancou A & 200 & 0 & 0,0 & $21(16)$ & 9,5 & 221 & - & - & - & - \\
\hline Troubat, C. $7 \mathrm{a}$ & 1012 & 2 & 0,2 & $11(9)$ & 1,1 & 1025 & - & - & - & - \\
\hline La Margineda, C. 8 & 1485 & 0 & 0,0 & $1(0)$ & 0,1 & 1486 & 2 & 1488 & 0,1 & 228 \\
\hline La Margineda, C. 7-6 & 1491 & 0 & 0,0 & $7(0)$ & 0,5 & 1498 & 2 & 1500 & 0,1 & 11 et 478 \\
\hline Troubat, C. 6 & 612 & 0 & 0,0 & $1(0)$ & 0,2 & 613 & 131 & 744 & 17,6 & - \\
\hline La Tourasse, D1 à D9 & 1454 & 0 & 0,0 & $14(3)$ & 1,0 & 1468 & 0 & 1468 & 0,0 & - \\
\hline Dufaure, C. 3 & 795 & 0 & 0,0 & $6(2)$ & 0,8 & 801 & 16 & 817 & 2,0 & 0 \\
\hline Dourgne, C. 9 & 171 & 0 & 0,0 & $19(0)$ & 10,0 & 190 & 0 & 190 & 0,0 & 0 \\
\hline La Margineda, C. 5 & 235 & 0 & 0,0 & $3(0)$ & 1,3 & 238 & 0 & 238 & 0,0 & $12(+11 \mathrm{C} 6 / 5)$ \\
\hline Le Bignalats, Cbg-ns & 152 & 0 & 0,0 & $22(11)$ & 12,6 & 174 & 0 & 174 & 0,0 & 0 \\
\hline Troubat, C. 5 & 400 & 0 & 0,0 & 0 & 0,0 & 400 & - & - & - & - \\
\hline La Margineda, C. 4 & 1324 & 0 & 0,0 & $12(0)$ & 0,1 & 1336 & 0 & 1336 & 0,0 & 78 \\
\hline
\end{tabular}

Tabl. 3 - Représentation des grands mammifères, des poissons et des oiseaux dans les échantillons étudiés. L'effectif entre parenthèses dans la colonne NR Carnivores correspond au NR des renards (Vulpes vulpes et/ou Alopex lagopus). 


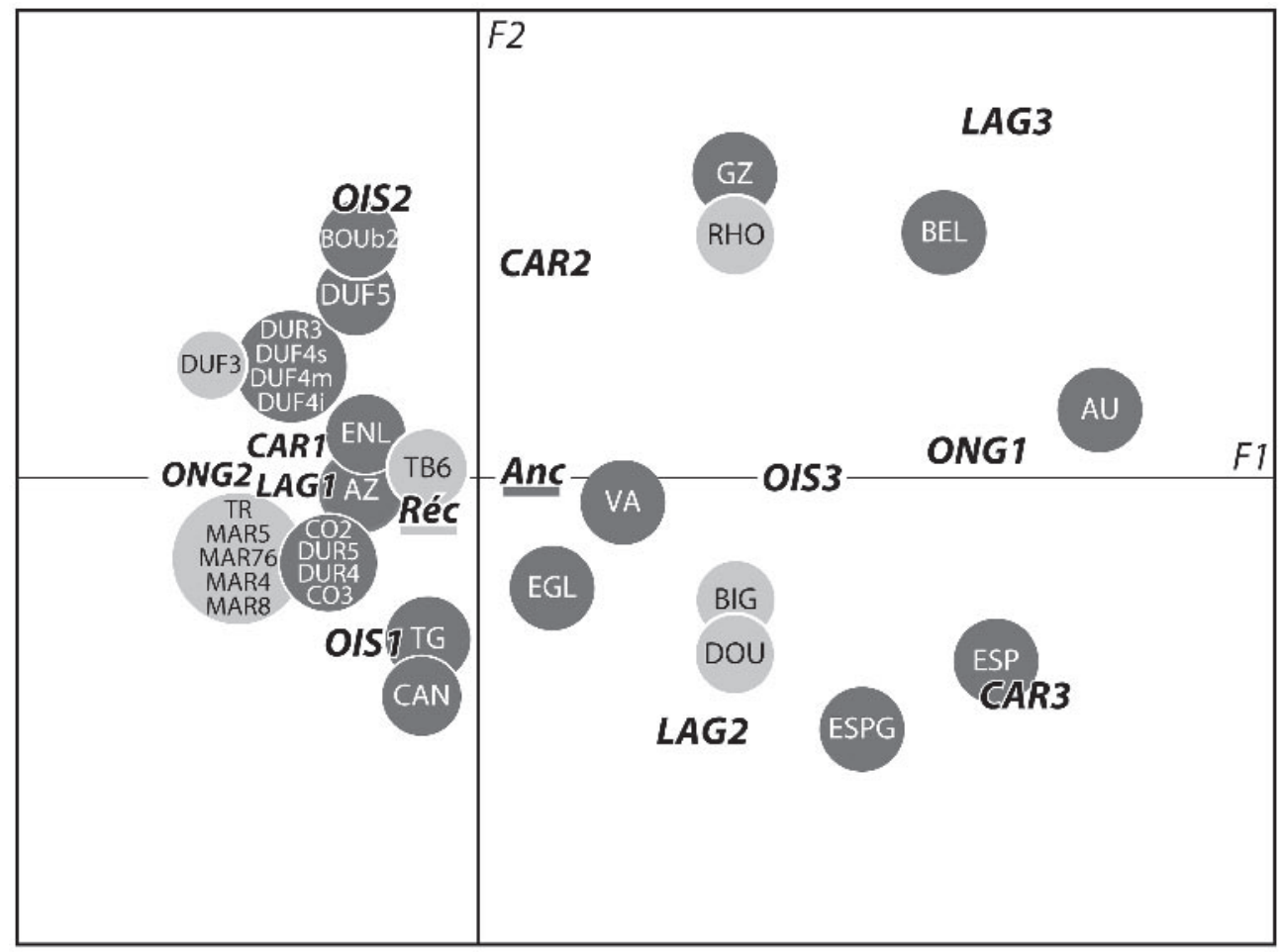

Fig. 6 - Des petits gibiers indifféremment chassés dans les phases ancienne et récente. Plan 1/2 de l'analyse factorielle des correspondances multiples montrant les liens entre échantillons et animaux chassés répartis en quatre groupes, Ongulés, Carnivores, Lagomorphes et Oiseaux. La variable Ongulés possède deux modalités qualitatives (ONG1 et ONG2), les variables Carnivores, Lagomorphes et Oiseaux en possèdent trois (CAR1 à CAR3, LAG1 à LAG3 et OIS1 à OIS3). La variable âge (variable supplémentaire) a deux modalités selon que les échantillons ont un âge antérieur à $12300 \mathrm{BP}$ (Anc.) ou postérieur (Réc.). Les échantillons anciens apparaissent en caractères blancs sur fond sombre, les échantillons récents en caractères noirs sur fond clair.

\section{UNE DIVERSIFICATION DES GIBIERS?}

Une fois démontré que la diversité des spectres de mammifères chassés n'a pas augmenté entre le Dryas ancien et le Boréal, intéressons-nous à la diversification éventuelle de l'ensemble des ressources animales.

Analyser les données en comparant les fréquences des mammifères (répartis en trois catégories : ongulés, carnivores et lagomorphes) à celles des oiseaux et des poissons est une opération irréalisable. L'information relative aux restes de poissons est trop partielle pour être prise en compte dans les calculs (tabl. 3). Les données relatives aux oiseaux sont quant à elles plus complètes mais elles sont hétérogènes en termes d'unité de décompte ${ }^{8}$. L'analyse des correspondances mutiples (ACM) permet de surmonter ce problème par la transformation de chaque variable quantitative en modalités qualitatives. Nous pouvons ainsi analyser conjointement quatre des cinq variables initiales (ongulés, carnivores, lagomorphes et oiseaux). Nous avons donné à une cinquième variable, l'âge des échantillons, un rôle de variable supplémentaire. L'analyse du tableau de Burt, construit en préalable à l'ACM, montre qu'aucun des tableaux de contingence croisant les modalités ancien et récent de la variable âge avec les différentes modalités des variables ongulés, carnivores, lagomorphes et oiseaux ne permet de mettre en évidence un lien significatif entre l'âge et la plus ou moins grande abondance relative de ces animaux. C'est bien ce qui apparaît également, de façon plus imagée, sur le premier plan factoriel de l'ACM (fig. 6). Sur le premier axe s'opposent les échantillons qui contiennent une forte proportion d'ongulés (côté négatif) à ceux dans lesquels lagomorphes, oiseaux et carnivores sont abondants (côté positif). De plus, il apparaît clairement que le tri des différents échantillons ne se fait pas selon leur âge. Plus encore, la projection des modalités ancien et récent de la variable binaire âge (introduite en variable supplémentaire) étant proche de l'origine des axes, il n'est pas possible d'envisager l'existence d'un lien avec aucune autre modalité. En d'autres termes, les petits animaux n'étaient pas davantage chassés dans la phase récente que dans la phase ancienne : il existe au cours des deux périodes des sites dans lesquels les ongulés furent les proies majoritaires, et d'autres dans lesquels carnivores, lagomorphes ou oiseaux furent capturés en grand nombre. Les données ne documentent donc pas l'a priori d'une intensification de la chasse aux petits gibiers entre la fin du Tardiglaciaire et le début du Postglaciaire. Un tel résultat était d'ailleurs prévisible puisque les deux seuls sites des Pyrénées françaises où les oiseaux ont été acquis en très grand nombre (la Vache et les Églises) n'appartiennent pas à la phase récente ${ }^{9}$. Si l'on prend en considération la grotte de Belvis, qui compte également de 
nombreux restes aviaires (mais dans une moindre mesure que les deux sites précédents), on s'aperçoit que ce petit secteur d'altitude de la haute Ariège comprend des sites où le Bouquetin, le Lagopède (Lagopus sp.) et le Lièvre variable (Lepus timidus) ont été recherchés (fig. 7). De plus, c'est uniquement dans les sites de ce secteur (cf. tabl. 3) que la fréquence des restes de poissons quitte le domaine de l'anecdotique, autour de 12300 BP (Belvis ou les Églises) et également plus au sud à la même altitude, vers 10500 BP (Balma de la Margineda). La chasse aux oiseaux, plus particulièrement aux lagopèdes, est donc, dans l'état actuel des données, un phénomène associé à des occupations de moyenne altitude liées à une acquisition saisonnière (Delpech et Le Gall, 1983; Fontana, 1998a et 1999; Pailhaugue, 2003) de gibiers spécifiques, qu'ils soient grands ou petits. Il s'agit donc d'un phénomène local et en aucun cas du témoignage d'une évolution de la chasse aux oiseaux qui aurait débuté au Dryas ancien pour se poursuivre jusqu'à la fin du Magdalénien. Cette particularité locale est d'autant plus ponctuelle qu'elle ne perdure pas, comme en témoignent les données des sites aziliens et mésolithiques. Le constat est identique pour les Lagomorphes. D'une part, l'acquisition de nombreux lièvres variables à Gazel est une exception, de surcroit hors massif pyrénéen et au début du Dryas ancien. D'autre part, si le Lièvre variable fut capturé dans les sites de la haute Ariège, sa part reste très minoritaire (cf. tabl. 3), sans commune mesure avec celle, bien plus importante (et qui perdurera), qui est documentée dans les sites équivalents des Alpes du nord (Bridault et Chaix, 2002) à cette période. La chasse au Lièvre variable ne sera d'ailleurs pas remplacée par la chasse au Lièvre commun (Lepus europaeus), à la différence des sites alpins de l'Italie du Nord-Est (voir Bridault et Chaix, ibid.). Quant au Lapin (Oryctolagus cuniculus), il n'est qu'un gibier anecdotique, quelle que soit la période envisagée. Les carnivores, dont la part excède $10 \%$ dans cinq cas seulement, sont représentés en très grande majorité par les renards (le plus souvent le Renard roux, Vulpes vulpes) dont l'origine anthropique n'est pas toujours démontrée (notamment à Aurensan et aux Espélugues où ils sont particulièrement bien représentés). La rareté des Mustélidés et du Chat sauvage (Felis sylvestris) dans les sites du domaine pyrénéen ${ }^{10} \mathrm{~s}$ 'oppose à leur importance dans les sites du Jura et des Alpes du nord dès la fin du Tardiglaciaire (Bridault et Chaix, ibid.).

\section{CONCLUSIONS}

L'analyse des 56 séries fauniques issues de 21 sites pyrénéens a permis de répondre aux trois questions posées, relatives à une éventuelle évolution de la prédation à la fin du Tardiglaciaire. L'étude de l'évolution des gibiers entre 15000 et $8000 \mathrm{BP}$ confirme que le Renne n'était plus chassé dès 12300 BP. Le Cerf et le Bouquetin, qui deviennent les deux gibiers principaux, selon les secteurs, étaient d'ailleurs déjà davantage chassés, dès $13000 \mathrm{BP}$ pour le Cerf et dès $12500 \mathrm{BP}$ pour le Bouquetin. L'analyse de la diversité des spectres de mammifères a démontré qu'elle n'avait pas évolué au cours du temps. Enfin, l'absence de toute augmentation significative de la part des petits gibiers réfute l'existence d'une diversification de la prédation.

L'analyse de ces données nous permet-elle de mettre en évidence d'autres tendances?

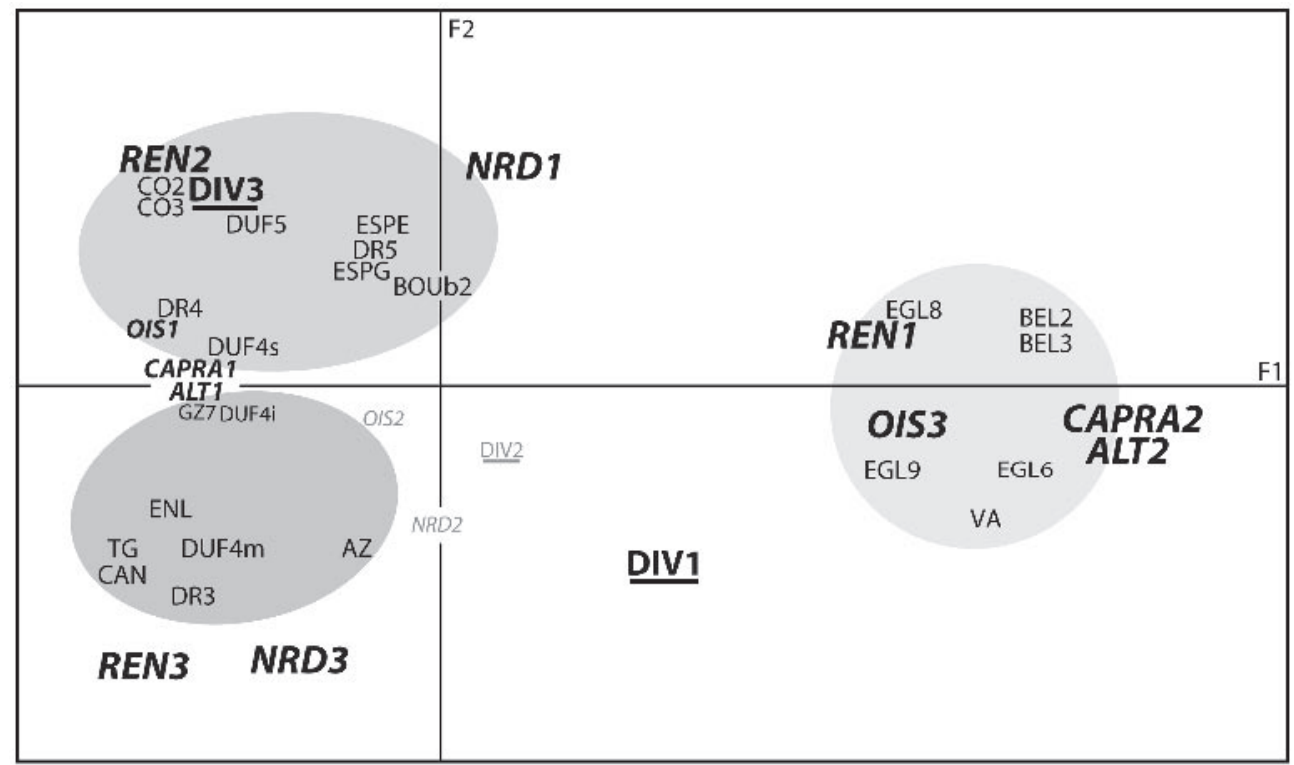

Fig. 7 - Plan 1/2 de l'analyse factorielle des correspondances multiples croisant les échantillons de la phase ancienne pour lesquels la totalité de l'information est disponible avec les variables : fréquences relatives de Renne (REN, 3 modalités), de Bouquetin (CAPRA, 2 modalités) et d'oiseaux (OIS, 3 modalités), altitude (ALT, 2 modalités, $\leq 500 \mathrm{~m}$ et $>500 \mathrm{~m}$ ) et diversité (DIV, entropie de Shannon-Wiener, 3 modalités, variable supplémentaire). Les modalités des variables en grisé sont mal représentées dans ce plan. Les échantillons sont rassemblés en trois groupes (plages grises) suivant les résultats d'une classification ascendante hiérarchique construite à partir de leurs coordonnées dans l'espace des trois premiers facteurs. 


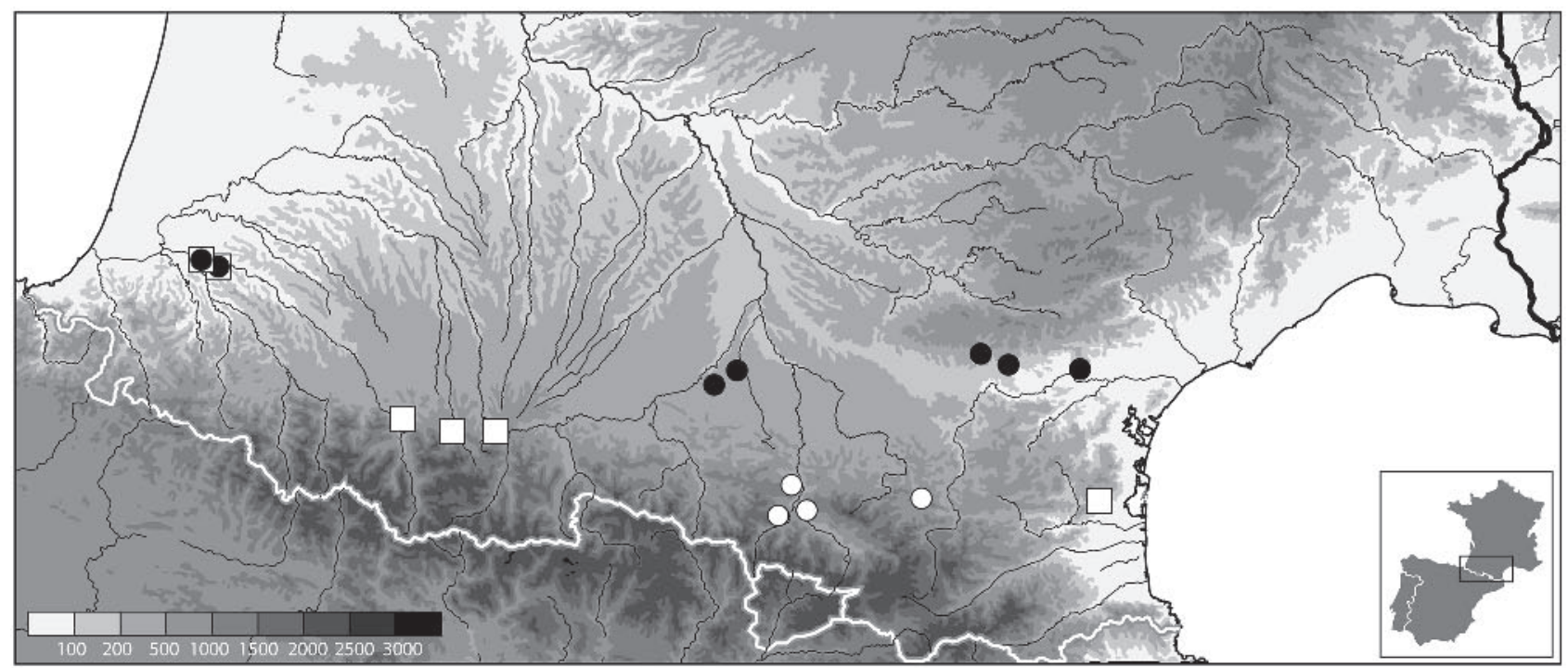

Fig. 8 - Distribution spatiale des échantillons de la phase ancienne scindés en trois groupes selon les résultats de l'ACM présentée sur la figure 7. Ronds noirs : échantillons riches, peu diversifiés à Renne dominant; ronds blancs : échantillons à Bouquetin et Oiseaux; carrés blancs : échantillons pauvres très diversifiés.

L'étude des séries antérieures à $12300 \mathrm{BP}$ a souligné la forte opposition entre les sites d'altitude à bouquetins-oiseaux et les sites de piémont où le Renne est mieux représenté, les bouquetins et oiseaux plus rares. Elle distingue également, parmi les sites de piémont, ceux dont les échantillons sont de grande taille, peu diversifiés et dominés par le Renne, de ceux qui sont très diversifiés et de petite taille (cf. fig. 7). Si l'on replace ces trois groupes de sites anciens sur la carte de la chaîne pyrénéenne et de son piémont septentrional, on constate qu'ils se répartissent en trois zones géographiques (fig. 8). Les sites qui ont livré de grands échantillons peu diversifiés dans lesquels le Renne est très abondant ne se trouvent jamais en deçà d'une ligne qui joint la montagne Noire aux Landes. Ceux d'où proviennent de petits échantillons très diversifiés à Renne moyennement abondant occupent un secteur plus méridional, majoritairement occidental. Quant aux sites d'altitude, aux échantillons plus ou moins riches et diversifiés, ils occupent, dans l'état actuel des connaissances, une région encore plus méridionale et orientale. Or, on s'aperçoit que le premier et le dernier groupe renvoient à deux réalités distinctes: les sites de piémont qui témoignent de chasses presque exclusives au Renne sont systématiquement de «grands sites» (Gazel, Canecaude, Tournal, Enlène, le Mas d'Azil, Dufaure $4 \mathrm{~m}$, 4i et Duruthy 3) occupés en partie au Dryas ancien et en saison froide, alors que les sites orientaux plus en altitude, plus ou moins vastes (Belvis, la Vache, les Églises), sont situés entre 12400 BP et 12300 BP et sont occupés durant la belle saison (Fontana, 1998a et 1999). Cela indique également que cette configuration d'importantes installations hivernales associées à la chasse au Renne disparaît peu avant $13000 \mathrm{BP}$, sans que l'on sache précisément ce qui lui succède. Les rares occupations postérieures, dans ce secteur, semblent bien moins importantes (Sacchi, 1986) et témoignent d'une modification, qui reste à caractériser, dans l'exploitation des ressources et dans l'habitat. Ce phénomène témoigne-t-il du début d'un retrait progressif du Renne dès $13000 \mathrm{BP}$, comme pourrait l'indiquer l'augmentation de la part du Cerf dans certains secteurs à cette même date? Malheureusement, les seules données fiables concernant le Bølling sont celles des sites d'altitude, très localisés, occupés à partir de $12400 \mathrm{BP}$, dont on ignore dans quelle mesure ils sont représentatifs de nouveaux choix. Soulignons enfin que notre démonstration de l'absence d'une diversification de la prédation dans les Pyrénées françaises à la fin du Tardiglaciaire confère un caractère plus général aux résultats obtenus pour l'Est de la France.

Remerciements : Nous exprimons toute notre gratitude à François-Xavier Chauvière, Nathalie DesseBerset, Armelle Gardeisen et Anne-Marie Moigne pour les informations inédites transmises, ainsi qu'à Christophe Delage pour la relecture du résumé en anglais. 
1978 et 1979). Les deux dates sont pourtant clairement incompatibles lorsque l'on met en regard BOR 6 avec l'intervalle à $1 \sigma$ obtenu par la calibration de LY 858 (13222-12894 BP cal). La date TL correspond à un âge ${ }^{14} \mathrm{C}$ peu antérieur à $10000 \mathrm{BP}$.

(4) Si l'ajout d'un ou deux taxons à fréquences relatives faibles à un échantillon ne modifie guère l'entropie de Shannon-Wiener, il en va tou autrement de la régularité puisque celle-ci est le rapport de l'entropie à l'entropie maximale (qui n'est autre que le logarithme du nombre de taxons représentés dans l'échantillon).

(5) Que ce soit l'indice de Shannon-Wiener, l'indice de richesse de Margalef ou le contesté (Grayson, 1984), et cependant très utile, estimateur de la richesse spécifique (diversité alpha) obtenu par les techniques de raréfaction (Raup, 1975; Tipper, 1979; Legendre et Legendre, 1984; Gotelli et Colwell, 2001; Hammer et Harper, 2006).
(6) Le calcul du nombre normalisé de taxons dans chaque échantillon par raréfaction aboutit au même constat. Il montre clairement que les valeurs moyennes du nombre de taxons (R100) qui seraient représentés dans des échantillons de 100 restes ne sont pas différentes entre les deux périodes (Rphase $1=5,39$, Rphase $2=5,80 ; \mathrm{F}, \mathrm{p}=0,539 ; \mathrm{t}, \mathrm{p}=0,514$ ). (7) Mann-Whitney U, p = 0,352; Kolmogorov-Smirnov, $\mathrm{p}=0,674$.

(8) Les décomptes des séries de Gazel, Canecaude, Tournal et Belvis sont présentés en NMI (Vilette, 1983) alors que ceux de la majorité des autres séries publiées le sont en NR.

(9) S'il s'avérait que les restes aviaires issus des fouilles récentes de la grotte du Bourrouilla étaient nombreux, ils correspondraient à une période légèrement postérieure à $12300 \mathrm{BP}$ (cf. supra) et ne remettraient pas en question nos résultats.

(10) Le site mésolithique de Dourgne constitue une exception avec 17 restes de Martre (cf. tabl. 2).

\section{RÉFÉRENCES BIBLIOGRAPHIQUES}

ALTUNA J., MARIEZKURRENA K. (1995) - Les restes osseux de macromammifères, in L.G Straus dir., Les derniers chasseurs du monde pyrénéen. L'abri Dufaure: un gisement tardiglaciaire en Gascogne, Mémoire de la Société préhistorique française, t. XXII, Paris, p. 181-211.

ALTUNA J., MARSAN G. (1986) - Le gisement préhistorique de la grotte du Bignalats à Arudy (Pyrénées-Atlantiques). Première partie Présentation des fouilles et étude de la faune de mammifères, Archéologie des Pyrénées occidentales, t. 6, p. 53-73.

BOCHERENS H., DRUCKER D., BILLIOU D., MOUSSA I. (2005) Une nouvelle approche pour évaluer l'état de conservation de l'os et du collagène pour les mesures isotopiques (datation au radiocarbone, isotopes stables du carbone et de l'azote), L'Anthropologie, t. 109, $n^{\circ} 3$, p. $557-567$

BOUCHUD J. (1972) - L'avifaune des grottes d'Aurensan et de Lourdes (Hautes-Pyrénées), Bulletin de l'Association française pour l'étude du Quaternaire, t. 1, p. 51-58.

BRIDAULT A. (1994) - Les économies de chasse épipaléolithiques et mésolithiques dans le Nord et l'Est de la France : nouvelles analyses, Anthropozoologica, t. 19, p. 55-67.

BRIDAULT A. (1997) - Broadening and diversification of hunted resources from the Late Palaeolithic to the Late Mesolithic in the North and East of France and bordering areas, Anthropozoologica, t. $25-26$, p. $295-308$

BRIDAULT A., CHAIX L. (2002) - Ruptures et équilibres dans la grande faune à la fin du Pléistocène et durant l'Holocène ancien en Europe occidentale, Equilibres et ruptures dans les écosystèmes depuis 20000 ans en Europe de l'Ouest, Actes du colloque international de Besançon, Presses universitaires franc-comtoises, Besançon.

BRIDAULT A., FONTANA L. (2003) - Enregistrement des variations environnementales par les faunes chassées, dans les zones de moyenne montagne d'Europe occidentale, du Tardiglaciaire au début de l'Holocène, Le rôle de l'environnement dans les comportements alimentaires des chasseurs-cueilleurs, Actes du XIV congrès UISPP, session 3 Paléoécologie, université de Liège, 2001, BAR International Series, 1105, Oxford, p. 55-65.

BRIDAULT A., CHAIX L., PION G., OBERLIN C., THIÉBAULT S., ARGANT J. (2000) - Position chronologique du Renne (Rangifer tarandus L.) à la fin du Tardiglaciaire dans les Alpes du nord françaises et le Jura méridional, in G. Pion dir., Le Paléolithique supérieur récent : nouvelles données sur le peuplement et l'environnement, Actes de la table ronde de Chambéry, 1999, Mémoire de la Société préhistorique française, t. XXVIII, Paris, p. 47-57.

CLOT A. (1983) - Déterminations de paléontologie quaternaire dans le bassin de l'Adour (première série), Cahiers du Groupe archéologique des Pyrénées occidentales, t. 3, p. 121-139.

CLOT A. (1984) - Faune de la grotte préhistorique du bois du Cantet (Espèche, Hautes-Pyrénées, France), Munibe, t. 36, p. 33-50.
CLOTTES J., DELPORTE H. (2003) - La grotte de la Vache (Ariège). I-Les occupations du Magdalénien, Réunion des musées nationaux et CTHS, Paris, $407 \mathrm{p}$.

COSTAMAGNO S. (2004) - Annexe 1 : Rapport de l'étude des ensembles osseux mis au jour, campagnes de fouilles 2002 à 2004, in M. Dachary, F.-X. Chauvière, S. Costamagno, L. Daulny, L. Detrain et C. Ferrier dir., La grotte de Bourrouilla (Pyrénées-Atlantiques), DFS de fouille programmée, service régional de l'Archéologie d'Aquitaine, p. 42-54.

COSTAMAGNO S. (2005) - Mobilité, territoires de chasse et ressources animales au Magdalénien final en contexte pyrénéen : le niveau 7a de la grotte-abri du Moulin (Troubat, Hautes-Pyrénées), in J. Jaubert et M. Barbaza dir., Territoires, déplacements, mobilité, échanges, Actes du $126^{e}$ congrès national des Sociétés historiques et scientifiques, Toulouse, 2001, CTHS, Paris, p. 371-383.

COSTAMAGNO S, COCHARD D., FERRIE J-G, LAROULANDIE V., CAZALS N., LANGLAIS M., VALDEYRON N., DACHARY M., BARBAZA M., GALOP D., MARTIN H., PHILIBERT S. (2008) - Nouveaux milieux, nouveaux gibiers, nouveaux chasseurs? Évolution des pratiques cynégétiques dans les Pyrénées du Tardiglaciaire au début du Postglaciaire, Bulletin de la Société préhistorique française, t. $105, \mathrm{n}^{\circ} 1$, p. 17-27.

DAGET J. (1976) - Les modèles mathématiques en écologie, Collection d'Écologie, $\mathrm{n}^{\circ}$ 8, Masson, $172 \mathrm{p}$.

DELPECH F. (1978) - Les faunes magdaléniennes et aziliennes du gisement de Duruthy à Sorde-L'Abbaye (Landes), in R. Arambourou dir., Le gisement préhistorique de Duruthy à Sorde-L'Abbaye (Landes). Bilan des recherches de 1958 à 1975, Mémoire de la Société préhistorique française, t. XIII, Paris, p. 110-116.

DELPECH F. (1981) - La faune magdalénienne de la salle des Morts à Enlène, Montesquieu-Avantès (Ariège), in R. Bégouen et J. Clottes dir., Nouvelles fouilles dans la salle des Morts de la caverne d'Enlène à Montesquieu-Avantès (Ariège), XXI congrès préhistorique de France, Montauban-Cahors, 1979, vol. 1, Société préhistorique française, Paris, p. 65-69.

DELPECH F. (1983) - Les faunes du Paléolithique supérieur dans le Sud-Ouest de la France, Cahiers du Quaternaire, ${ }^{\circ}$ 6, CNRS, Paris, $453 \mathrm{p}$.

DELPECH F., LE GALL O. (1983) - La faune magdalénienne de la grotte des Églises (Ussat, Ariège), Préhistoire ariègeoise, t. XXXVIII, p. 91-118.

DESSE-BERSET N. (inédit) - Les poissons de la grotte Gazel (couche 7, Magdalénien moyen).

DRUCKER D. (2001) - Validation méthodologique de l'analyse isotopique d'ossements fossiles et apports aux reconstitutions paléoécologiques du Paléolithique supérieur du Sud-Ouest de la France, thèse de doctorat, université de Paris VI, Paris, 222 p. 
EASTHAM A. (1995) - L'écologie avienne, in L.G Straus dir., Les derniers chasseurs du monde pyrénéen. L'abri Dufaure : un gisement tardiglaciaire en Gascogne, Mémoire de la Société préhistorique française, t. XXII, Paris, p. 219-233.

EASTHAM A. (1998) - Magdalenians and snowy owls: bones recovered at the Grotte de Bourrouilla (Arancou, Pyrénées-Atlantiques), Paléo, t. 10 , p. $95-107$.

EASTHAM A. (1999) - Les oiseaux et la microfaune, in C. Chaucha dir., L'habitat magdalénien de la grotte du Bourrouilla à Arancou (Pyrénées-Atlantiques), Gallia Préhistoire, t. 41, p. 113-127.

ÉVIN J. (1978) - Fiches de résultats d'échantillons soumis au datage par le radiocarbone, in $\mathrm{R}$. Arambourou dir., Le gisement préhistorique de Duruthy à Sorde-L'Abbaye (Landes). Bilan des recherches de 1958 à 1975, Mémoire de la Société préhistorique française, t. XIII, Paris, p. 127.

ÉVIN J. (1995) - Les datations radiocarbone in L.G. Straus dir., Les derniers chasseurs de rennes du monde pyrénéen, Mémoire de la Société préhistorique française, t. XXII, p. 29-32.

FONTANA L. (1998a) - Subsistance et territoire au Magdalénien supérieur dans les Pyrénées : l'apport des données archéozoologiques de la grotte de Belvis (Aude),Bulletin Préhistoire du Sud-Ouest, Nouvelles Études, t. 5, n² 2, p. 131-146.

FONTANA L. (1998b) - Mobilité et subsistance au Magdalénien dans le Languedoc occidental et le Roussillon, thèse de doctorat, université de Paris I-Panthéon-Sorbonne, Paris, 287 p.

FONTANA L. (1999) - Mobilité et subsistance au Magdalénien dans le bassin de l'Aude, Bulletin de la Société préhistorique française, t. 96, $\mathrm{n}^{\circ} 2$, p. 175-190.

FOSSE P. (1999) - La grande faune mammalienne : remarques préliminaires, in $\mathrm{C}$. Chauchat dir., L'habitat magdalénien de la grotte du Bourrouilla à Arancou (Pyrénées-Atlantiques), Gallia Préhistoire, t. 41 , p. $98-113$.

GARDEISEN A. (2008) - La fauna de mamifers dels nivells azilians de la Balma de la Margineda, in J. Guilaine, M. Barbaza et M. Martzluf dir., Les excavacions a la Balma de la Margineda (1979-1991), vol. 4. Ministeri d'Afers Social Cultura Govern d'Andorra ed., Principat d'Andorra, p. 492-522.

GARDEISEN A. (sous presse) - La chasse à la Balma de la Margineda in J. Guilaine et M. Martzluff dir., Les excavacions a la Balma de la Margineda (1979-1991), vol. 5, Ministeri d'Afers Socials i Cultura Govern d'Andorra ed., Principat d'Andorra.

GEDDES D. (1993) - La faune (couche 9 de Dourgne, Aude), Dourgne : derniers chasseurs-collecteurs et premiers éleveurs de la haute vallée de l'Aude, Centre d'anthropologie des sociétés rurales/Archéologie en terre d'Aude, Toulouse/Carcassonne, p. 95-96.

GOTELLI N. J., COLWELL R.K. (2001) - Quantifying biodiversity: procedures and pitfalls in the measurement and comparison of species richness, Ecology Letters, vol. 4, p. 379-391.

GOURDAIN L. (2000) - Étude de la faune de l'ensemble D de la Tourasse (Saint-Martory, Haute-Garonne) : perspectives environnementales, mémoire de DEA, université de Paris I, Paris, 70 p.

GRAYSON D.K. (1984) - Quantitative Zooarchaeology, Studies in archaeological Science, Academic Press, 202 p.

HAMMER Ø., HARPER D. (2006) - Palaeontological data analysis, Blackwell Publishing, $351 \mathrm{p}$.

LAROULANDIE V. (1998) - Études archéozoologique et taphonomique des lagopèdes des saules de la grotte magdalénienne des Églises (Ariège), Anthopozoologica, t. 28, p. 45-54.

LAROULANDIE V. (2000) - Taphonomie et archéozoologie des oiseaux en grotte: applications aux sites paléolithiques du Bois Ragot (Vienne), de Combe Saunière (Dordogne) et de la Vache (Ariège), thèse de doctorat, université de Bordeaux I, Bordeaux, $396 \mathrm{p}$.
LAROULANDIE V. (2007) - Les restes aviaires des niveaux aziliens de la grotte-abri du Moulin (Troubat, Hautes-Pyrénées) : paléoenvironnements et modalités d'exploitation, Bulletin Préhistoire du Sud-Ouest, t. $14, \mathrm{n}^{\circ} 1$, p. 19-29.

LE GALL O. (1995a) - Ichtyologie, in L.G Straus dir., Les derniers chasseurs du monde pyrénéen. L'abri Dufaure: un gisement tardiglaciaire en Gascogne, Mémoire de la Société préhistorique française, t. XXII, Paris, p. 247-248.

LE GALL O. (1995b) - Les poisons de la Balma de la Margineda. Néolithique et Mésolithique (couches 3 à 6), in J. Guilaine dir., Les excavacions a la Balma de la Margineda (1979-1991), Govern d'Andorra, Andorra, p. 106-110.

LE GALL O. (1999a) - Les poissons, in C. Chauchat dir., L'habitat magdalénien de la grotte du Bourrouilla à Arancou (PyrénéesAtlantiques), Gallia Préhistoire, t. 41, p. 29-32.

LE GALL O. (1999b) - Éléments de réflexion sur la pêche dans le Bassin méditerranéen nord-occidental pendant le développement des faciès leptolithiques, in D. Sacchi dir., Les faciès leptolithiques du NordOuest méditerranéen : milieux naturels et culturels, Actes du XXIV congrès préhistorique de France, Carcassonne, 1994, vol 1., Société préhistorique française, Paris, p. 251-265.

LE GALL O. (2003) - Des Magdaléniens et... des poissons, in S. Costamagno et V. Laroulandie dir., Mode de vie au Magdalénien : apports de l'archéozoologie, Actes du XIV congrès UISPP, Liège, Symposium 6.4, BAR International Series, 1144, Oxford, p. 119-128.

LE GALL O. (2008) - Els peixos de la Balma de la Margineda (Capes de la 7 a la 10), in J. Guilaine, M. Barbaza et M. Martzluff dir., Les excavacions a la Balma de la Margineda (1979-1991), vol. 4, Ministeri d'Afers Social Cultura Govern d'Andorra ed., Principat d'Andorra, p. 532-539

LEGENDRE L., LEGENDRE P. (1984) - Écologie numérique, Masson, 2 t., 197 et $247 \mathrm{p}$

LEGENDRE L., LEGENDRE P. (1998) - Numerical Ecology, second english edition, Developments in Environmental Modelling, 20 Elsevier, $853 \mathrm{p}$

MAGURRAN A.E. (1988) - Ecological Diversity and its Measurement Princeton University Press, 179 p.

MARTIN H. (1994) - Nouveaux milieux, nouveaux chasseurs, une approche des comportements au Postglaciaire à travers l'étude des saisons de capture du gibier, thèse de doctorat, université de Toulouse 2-Le Mirail, Toulouse, 383 p.

MOIGNE A.-M. (2003) - Les faunes de grands mammifères de la grotte des Conques à Vingrau, Les Conques. Des chasseurs magdaléniens et leur territoire, ERAUL, t. 101, Liège, p. 75-103

OMNES J. (1984) - Le locus I des Espélugues de Lourdes (HautesPyrénées). Un petit habitat magdalénien de profondeur, Préhistoire ariégeoise, t. XXXIX, p. 85-101.

PAILHAUGUE N. (1995) - La faune de la salle Monique, grotte de la Vache (Alliat, Ariège), Bulletin de la Société préhistorique de l'Ariège, t. 50 , p. $225-289$.

PAILHAUGUE N. (2003) - La faune de la salle Monique, in J. Clottes et H. Delporte dir., La grotte de la Vache (Ariège), fouilles Romain Robert. I-Les occupations du Magdalénien, Réunion des musées nationaux et CTHS, Paris, p. 73-139.

PAQUEREAU M.-M. (1978) - Analyses palynologiques de l'abri Duruthy à Sorde-L'Abbaye (Landes), in R. Arambourou dir., Le gisement préhistorique de Duruthy à Sorde-L'Abbaye (Landes). Bilan des recherches de 1958 à 1975, Mémoire de la Société préhistorique française, t. XIII, Paris, p. 96-109.

PATOU M. (1984) - La faune de la galerie rive droite du Mas d'Azil (Ariège) : données paléoclimatiques et palethnographiques, Bulletin de la Société préhistorique française, t. 81, p. 311-319.

PATOU-MATHIS M., BAYLE G., PALETTA C. (1999) - Étude archéozoologique du niveau magdalénien «ancien» de la grotte Tournal à Bize (Aude, France), Préhistoire européenne, t. 14, p. 115-133. 
PEET R.K. (1974) - The Measurement of Species Diversity, Annual Review of Ecology and Systematics, vol. 5, p. 285-307.

PIELOU E.C. (1966) - The measurement of diversity in different types of biological collections, Journal of Theoretical Biology, vol. 13, p. 131-144.

RAUP D.M. (1975) - Taxonomic diversity estimation using rarefaction, Paleobiology, vol. 1, p. 333-342.

SACCHI D. (1986) - Le Paléolithique supérieur du Languedoc occidental et du Roussillon, éd. du CNRS, Paris, 284 p.

SCHVOERER M. (1978) - Datation absolue par thermoluminescence des niveaux magdaléniens IV et VI de l'abri Duruthy, in R. Arambourou dir., Le gisement préhistorique de Duruthy à Sorde-L'Abbaye (Landes). Bilan des recherches de 1958 à 1975, Mémoire de la Société préhistorique française, t. XIII, Paris, p. 128-130.

SCHVOERER M. (1979) - Datation absolue par thermoluminescence de minéraux brûlés. Application à des fragments de grès provenant de foyers des niveaux magdaléniens IV et VI de Sorde-L'Abbaye (Landes). Possibilités actuelles de la méthode, La fin des temps glaciaires en Europe, coll. internationaux CNRS, $\mathrm{n}^{\circ} 271$, CNRS, Paris, p. 15-19.

SHANNON C., WEAVER W. (1949) - The Mathematical Theory of Communication, University of Illinois Press, Urbana, 117 p.

SPELLERBERG I.F., FEDOR P.J. (2003) - A tribute to Claude Shannon (1916-2001) and a plea for more rigorous use of species richness, species diversity and the «Shannon-Wiener» Index, Global Ecology and Biogeography, vol. 12, 3, p. 177-179.

STRAUS L.G. (1995) - Descriptions et comparaisons archéologiques, in L.G Straus dir., Les derniers chasseurs du monde pyrénéen. L'abri Dufaure: un gisement tardiglaciaire en Gascogne, Mémoire de la Société préhistorique française, t. XXII, Paris, p. 75-105.
THIBAULT C. (1978) - Étude géologique du gisement de Duruthy (Landes), in R. Arambourou dir., Le gisement préhistorique de Duruthy à Sorde-L'Abbaye (Landes). Bilan des recherches de 1958 à 1975, Mémoire de la Société préhistorique française, t. XIII, Paris, p. 67-96.

TIPPER J.C. (1979) - Rarefaction and rarefaction - the use and abuse of a method in paleoecology, Paleobiology, vol. 5, 4, p. 423-434.

VILETTE P. (1983) - Avifaunes du Pléistocène final et de l'Holocène dans le Sud de la France et en Catalogne, Atacina, t. 11, Carcassonne, $190 \mathrm{p}$.

VILETTE P. (1994) - L'avifaune, in D. Sacchi, un site paléolithique supérieur de moyenne altitude dans les Pyrénées : la Cauna de Belvis (France), Human adaptations to the Mountain Environment in the Upper Palaeolithic and Mesolithic, Actes du colloque de Trento, Prehistoria Alpina, ${ }^{\circ}$ 28/2, p. 84-86.

\section{Laure FONTANA \\ LAMPEA (UMR 6636)}

Maison méditerranéenne des sciences de l'Homme

5, rue du Château-de-l'horloge BP 647, 13094 Aix-en-Provence Cedex 2 lfontana@mmsh.univ-aix.fr

Jacques Élie BROCHIER LAMPEA (UMR 6636)

Maison méditerranéenne des sciences de l'Homme

5, rue du Château-de-l'horloge

BP 647, 13094 Aix-en-Provence Cedex 2 jacques.brochier@univ-provence.fr 